To reference this article, please use the following citation:

Lawrie, J.B. (Forthcoming). Comments on a class of orthogonality relations relevant to fluid-structure interaction. Meccanica. DOI 10.1007/s11012-011-9471-8 


\title{
Comments on a class of orthogonality relations relevant to fluid-structure interaction
}

\author{
J.B. Lawrie \\ Department of Mathematics, Brunel University \\ Uxbridge, UB8 3PH, UK \\ jane.lawrie@brunel.ac.uk
}

Keywords: orthogonality relation; fluid-structure interaction; superposed fluids; acoustic silencer; flexible walls.

Abstract: This article presents an overview of the recent literature and summarises the major theoretical developments pertaining to a class of non-Sturm-Liouville orthogonality relations relevant to fluid-structure interaction.

\section{Introduction}

In two recent articles Amaouche and Meziani investigate the coupled frequencies of a hydroelastic system comprising two superposed fluids within a 2D container with one flexible wall $[1,2]$. The model problem and solution methods presented in the two articles are identical with the latter paper [2] offering more discussion but some of the same numerical results as the former [1]. In both articles the model problem is posed in terms of the fluid velocity potential. The fluid motions at the free surface and the fluid-fluid interface are described by the kinematic and dynamic boundary conditions and the normal component of fluid velocity is zero at the base of the container. Amaouche and Meziani express the solution as an eigenfunction expansion of the form

$$
\Phi(x, y, t)=e^{-i \omega t} \sum_{n=0}^{\infty} A_{n} Y_{n}(y) \cos \left(s_{n}(x-1)\right)+\text { c.c. }
$$

and state that the eigenfunctions $Y_{n}(y)$ satisfy a "new" orthogonality relation. Further, they refer to an earlier article [3] in which a similar but simpler orthogonality relation (OR) is presented.

In fact, the eigensystem satisfied by $Y_{n}(y)$ and $s_{n}, n=0,1,2, \ldots$ belongs to a well known class of non-Sturm-Liouville system for which ORs of the type presented in $[1,2]$ are typical. As far as the author is aware, that presented in [3] does appear to be the first appearance in the literature of this class of OR. In the intervening years, however, the theory underpinning this class of eigensystem has been extensively developed and has found application in many diverse model problems from the fields of structural acoustics and hydrodynamics. This article presents, for the Meccanica readership, an overview of the recent literature and summarises the major theoretical developments pertaining to this class of eigensystem. 


\section{An overview of recent developments}

From the author's point of view, the initial motivation for the development of ORs for waveguides with high order boundary conditions (such as those describing the motion of membranes or thin elastic plates) was the desire to solve problems involving acoustic scattering in waveguides involving two (or more) sections of duct each with different height and material property (see figure 1). For ducts with differing material properties but of the same height the Wiener-Hopf technique can provide a powerful solution method [4]. Whilst for duct sections of differing height but in which the underlying eigensystem is Sturm-Liouville in type, standard Fourier series provide solutions. Lawrie and Abrahams [5] recognised that there was a general class of boundary value problem involving either Helmholtz's or Laplace's equation together with high order boundary conditions for which the underlying eigensystem was nonSturm-Liouville but for which useful ORs existed. They were aware of and cited the Russian versions of two articles on the subject. It was thought that both dealt with explicit examples of such ORs but, whilst this is true of [6], it transpires that [7] does deal with some of the underlying theory. Lawrie and Abrahams were unfortunately not aware of the work presented in [3].

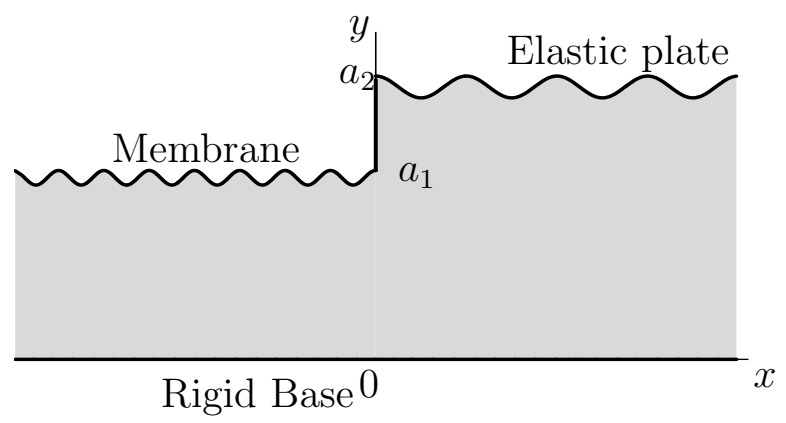

Figure 1: A duct configuration demonstrating change of height and material property.

It is worthwhile briefly summarising the main content of [5]. The article is concerned with the boundary value problem for an infinite duct with high order boundary conditions. Under the assumption of harmonic time dependence, such that the velocity potential may be expressed as

$$
\Phi(x, y, t)=\Re\left\{\phi(x, y) e^{-i \omega t}\right\}
$$

where $\omega=c k$ in which $c$ is the fluid sound speed and $k$ is the fluid wavenumber, the (non-dimensional) general boundary problem is posed in terms of Helmholtz's equation as

$$
\left\{\frac{\partial^{2}}{\partial x^{2}}+\frac{\partial^{2}}{\partial y^{2}}+1\right\} \phi(x, y)=0
$$

in which $x$ and $y$ are the usual Cartesian coordinates but are non-dimensionalised with respect to $k^{-1}$. The field equation holds in a strip of finite height $0 \leq y \leq a$ 
and infinite length $-\infty<x<\infty$ which is bounded by walls described by high-order boundary conditions. The mathematical statement of the most general, physically relevant, pair of boundary conditions is

$$
\mathcal{L}_{a}\left(\frac{\partial}{\partial x}\right) \frac{\partial \phi}{\partial y}+\mathcal{M}_{a}\left(\frac{\partial}{\partial x}\right) \phi=0, \quad y=a,
$$

on the upper waveguide surface together with

$$
\mathcal{L}_{0}\left(\frac{\partial}{\partial x}\right) \frac{\partial \phi}{\partial y}+\mathcal{M}_{0}\left(\frac{\partial}{\partial x}\right) \phi=0, \quad y=0,
$$

on the lower surface. Here $\mathcal{L}_{p}\left(\frac{\partial}{\partial x}\right)$ and $\mathcal{M}_{p}\left(\frac{\partial}{\partial x}\right), p=a, 0$, are differential operators of the form

$$
\mathcal{L}_{p}\left(\frac{\partial}{\partial x}\right)=\sum_{k=0}^{K_{p}} c_{k}^{p} \frac{\partial^{2 k}}{\partial x^{2 k}}, \quad \mathcal{M}_{p}\left(\frac{\partial}{\partial x}\right)=\sum_{j=0}^{J_{p}} d_{j}^{p} \frac{\partial^{2 j}}{\partial x^{2 j}},
$$

where $c_{k}^{0}, c_{k}^{a}, d_{j}^{0}, d_{j}^{a}$ are constant coefficients. Note that, for physical reasons, only even derivatives in $x$ are included. Higher derivatives in $y$ are easily removed by recourse to equation (3), hence the absence of such terms in (4) and (5). The velocity potential can be expressed as an eigenfunction expansion which, for wave propagation in the positive $x$ direction, takes the form

$$
\phi(x, y)=\sum_{n=0}^{\infty} A_{n} Y_{n}(y) e^{i s_{n} x}, \quad x>0
$$

where $A_{n}$ is the modal amplitude, the wavenumbers $s_{n}, n=0,1,2, \ldots$ are either real or have positive imaginary part and $Y_{n}(y)$ satisfies the eigensystem comprising

$$
Y_{n}^{\prime \prime}(y)=\gamma_{n}^{2} Y_{n}(y), \quad \gamma_{n}=\left(s_{n}^{2}-1\right)^{1 / 2}
$$

together with:

$$
\begin{aligned}
P_{a}\left(s_{n}\right) Y_{n}^{\prime}(a)+Q_{a}\left(s_{n}\right) Y_{n}(a) & =0 \\
P_{0}\left(s_{n}\right) Y_{n}^{\prime}(0)+Q_{0}\left(s_{n}\right) Y_{n}(0) & =0
\end{aligned}
$$

where, here and henceforth, the primes denote differentiation with respect to $y$. The functions $P_{p}(s)$ and $Q_{p}(s), p=a, 0$, are characteristic polynomials and correspond to the action of the operators $\mathcal{L}_{p}\left(\frac{\partial}{\partial x}\right)$ and $\mathcal{M}_{p}\left(\frac{\partial}{\partial x}\right)$ on the eigen-expansion (7). That is,

$$
P_{p}\left(s_{n}\right) \equiv \mathcal{L}_{p}\left(i s_{n}\right) \text { and } Q_{p}\left(s_{n}\right) \equiv \mathcal{M}_{p}\left(i s_{n}\right) .
$$

On solving (8) subject to (10), it is clear that $Y_{n}(y)$ may be expressed as:

$$
Y_{n}(y)=P_{0}\left(s_{n}\right) \cosh \left(\gamma_{n} y\right)-\frac{1}{\gamma_{n}} Q_{0}\left(s_{n}\right) \sinh \left(\gamma_{n} y\right) .
$$

The dispersion relation is then deduced using (12) together with (9). That is,

$$
\begin{aligned}
K(\gamma) & =\left[\gamma^{2} P_{0}(s) P_{a}(s)-Q_{0}(s) Q_{a}(s)\right] \frac{\sinh (\gamma a)}{\gamma} \\
& +\left[Q_{a}(s) P_{0}(s)-P_{a}(s) Q_{0}(s)\right] \cosh (\gamma a)=0,
\end{aligned}
$$


where $s^{2}=\gamma^{2}+1$. The fact that the operators (6) contain only even derivatives in $x$ ensures that the characteristic polynomials are functions of $s^{2}$. Thus, the dispersion relation can be expressed as a function of even powers of $\gamma$. The roots of $K(\gamma)=0$ have the following properties: i) for every root, $\gamma_{n}$, there is another root, $-\gamma_{n}$; ii) there is a finite number of real roots; iii) there is an infinite number of imaginary roots; iv) complex roots, $\pm \gamma_{c}$ and $\pm \gamma_{c}^{*}$ can occur. For real and imaginary roots, the convention is adopted that the $+\gamma_{n}$ roots are either positive real or have positive imaginary part. They are ordered sequentially, real roots first and then by increasing imaginary part. Thus, $\gamma_{0}$ is always the largest real root. For any complex root, say $\gamma_{c}$, lying in the upper half of the complex $\gamma$-plane, then minus the complex conjugate, $-\gamma_{c}^{*}$, also lies in this half plane. Such pairs are incorporated into the sequence of roots according to the magnitude of their imaginary part, and in the order $\gamma_{c}$ followed by $-\gamma_{c}^{*}$. It is assumed that no root is repeated.

Lawrie and Abrahams present the general OR satisfied by $Y_{n}(y), n=0,1,2, \ldots$ but, as may be expected, this is somewhat cumbersome. They then focus on the case where the lower boundary is rigid and the upper boundary comprises a membrane (as in the left hand side of figure 1). The eigensystem for this system is retrieved from (3)-(13) on putting $Q_{0}(s)=0, P_{0}(s)=1, P_{a}(s)=-s^{2}+\mu^{2}$ and $Q_{a}=\alpha$ where the quantities $\mu$ and $\alpha$ are defined in [5]. It transpires that the OR reduces to

$$
\alpha \int_{0}^{a} Y_{m} Y_{n} d y+Y_{m}^{\prime}(a) Y_{n}^{\prime}(a)=C_{m} \delta_{m n}
$$

where $\delta_{m n}$ is the Kronecker delta function and

$$
C_{m}=\left.\frac{Y_{m}^{\prime}(a)}{2 \gamma_{m}} \frac{d}{d \gamma} K(\gamma)\right|_{\gamma=\gamma_{m}},
$$

which is essentially that presented in [3]. The same OR was exploited in $[8,9]$ to address problems involving membrane bounded ducts with abrupt changes in height and/or material property. A similar OR was noted by Kaplunov et al [10].

Subsequent to the publication of [5], a number of authors utilised this approach to address a wide range of problems involving elastic plates or ice sheets floating on water of finite depth [11]-[18]. Further ORs of this class have been presented for waveguides in which one boundary comprises an elastic plate and the other a membrane or free surface $[19,20]$. In each of [11]-[20] the appropriate OR can be obtained directly from the general case presented in [5] by suitable choice of $P_{0}(s), P_{a}(s), Q_{0}(s)$ and $Q_{a}(s)$.

In the field of noise-control ORs of this class have been deduced for a range of prototype or canonical silencers [21]-[23]. In this case, due to the insertion of internal structures or linings into the waveguide (see figure 2), there is a subtle difference in the form of the ORs. The inclusion of a porous lining (modelled as an equivalent fluid layer in which the density and propagation constant are complex) or an internal structure such a membrane into the duct necessitates a piece-wise definition of $Y_{n}(y)$ :

$$
Y_{n}(y)= \begin{cases}Y_{1 n}(y), & 0 \leq y<d \\ Y_{2 n}(y), & d<y \leq a\end{cases}
$$



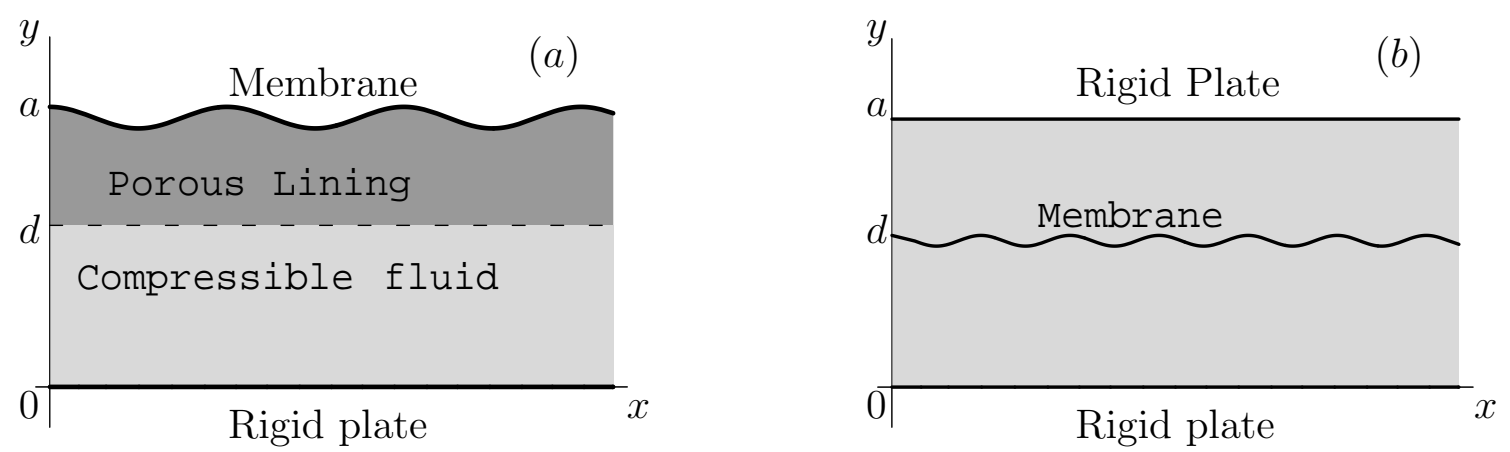

Figure 2: (a) A classic dissipative silencer and (b) the drum-like silencer: both demonstrating "fluid layering".

where $Y_{1 n}^{\prime}(d)=Y_{2 n}^{\prime}(d)$. The modified ORs are thus almost identical to that used in $[1,2]$. For example [21, 22], the eigenfunctions for the dissipative silencer shown in figure 2 (a) satisfy the OR:

$$
\alpha \int_{0}^{d} Y_{1 m} Y_{1 n} d y+\alpha \beta \int_{d}^{a} Y_{2 m} Y_{2 n} d y+\beta Y_{2 m}^{\prime}(a) Y_{2 n}^{\prime}(a)=\beta C_{m} \delta_{m n}
$$

where $\beta$ is the ratio of the density of the porous media to that of the acoustic fluid, $\alpha$ is the fluid loading parameter and $C_{m}$ is given by (15). The OR for the "drum-like" silencer $[22,23]$ of figure 2 (b) is similar in structure:

$$
\alpha \int_{0}^{d} Y_{1 m}(y) Y_{1 n}(y) d y+\alpha \int_{d}^{a} Y_{2 m}(y) Y_{2 n}(y) d y+Y_{n}^{\prime}(d) Y_{m}^{\prime}(d)=C_{n} \delta_{m n}
$$

in this case, of course, every occurrence of $Y_{m}^{\prime}(a)$ is replaced by $Y_{m}^{\prime}(d)$ since the membrane lies along $y=d$. Hydrodynamic applications that involve layered fluids $[24,25]$ and submersed plates [26] have also been addressed. Interestingly, the article by Mohapatra et al [25] considers a boundary value problem which is very similar to that of $[1,2]$ and uses the same OR. Further extensions include the development of the theory for wave propagation in 3D waveguides with flexible boundaries [27]-[28] and porous linings [29].

\section{Properties of a typical eigensystem}

The general class of eigensystem has well recognised properties, some of which are discussed in [7]. More recently Lawrie [19] has proven that the eigenfunctions are linearly dependent and has addressed the issue of point-wise convergence. The general properties as given in [19] are discussed here for a duct in which the lower boundary is rigid and the upper boundary comprises an elastic plate (as in the right hand side of figure 1). The dispersion relation and OR can be retrieved from (3)-(13) on taking $Q_{0}(s)=0, P_{0}(s)=1, P_{a}(s)=s^{4}-\mu^{4}$ and $Q_{a}=-\alpha$, where $\mu$ is the in 
vacuo plate wavenumber and $\alpha$ a fluid loading parameter; both quantities are defined in [28]. It is found that the eigenvalues $\gamma_{n}, n=0,1,2, \ldots$ are the roots of $K(\gamma)=0$ where

$$
K(\gamma)=\left\{\left(\gamma^{2}+1\right)^{2}-\mu^{4}\right\} Y^{\prime}(a)-\alpha Y(a)
$$

and that the eigenfunctions $Y_{m}(y), m=0,1,2, \ldots$ satisfy the OR

$$
\alpha \int_{0}^{a} Y_{m}(y) Y_{j}(y) d y+\left(\gamma_{m}^{2}+\gamma_{j}^{2}+2\right) Y_{j}^{\prime}(a) Y_{m}^{\prime}(a)=C_{j} \delta_{j m}
$$

where $C_{j}$ is given by (15). Note that, unlike (14), this cannot be expressed as a "true" inner product.

The eigenfunctions $Y_{j}(y), j=0,1,2, \ldots$ are linearly dependent:

$$
\sum_{n=0}^{\infty} \frac{Y_{n}^{\prime}(a) Y_{n}(y)}{C_{n}}=\sum_{n=0}^{\infty} \frac{\gamma_{n}^{2} Y_{n}^{\prime}(a) Y_{n}(y)}{C_{n}}=0
$$

where $0 \leq y \leq a$. Note that the number of linearly dependent sums is always equal to the number of corner or edge conditions that are required [19]. That is, the number of such sums is $M / 2$ where $M$ is the order of the highest derivative in the boundary condition (or in the case of two such boundaries, the sum of the orders). For a duct bounded above by an elastic plate and below by a rigid duct $M=4$ and thus, as indicated above, there are exactly two sums that express the linear dependence of the eigenfunctions. These expressions are especially important in the context of wave propagation in 3D ducts with flexible walls where they are used to ensure that the corner conditions are satisfied [28].

A Green's function can be constructed for the eigenfunctions:

$$
\alpha \sum_{n=0}^{\infty} \frac{Y_{n}(v) Y_{n}(y)}{C_{n}}=\delta(y-v)+\delta(y+v)+\delta(y+v-2 a), \quad-a \leq v, y \leq a
$$

where $\delta(y)$ is the usual Dirac delta function. This result is crucial to proving that the eigenfunction expansion representation of a suitably smooth function, say $f(y)$, converges point-wise to that function [19].

The eigenfunctions also satisfy the identities:

$$
\sum_{n=0}^{\infty} \frac{\left[Y_{n}^{\prime}(a)\right]^{2}}{C_{n}}=0, \quad \sum_{n=0}^{\infty} \frac{\gamma_{n}^{2}\left[Y_{n}^{\prime}(a)\right]^{2}}{C_{n}}=1
$$

Note that, in (21)-(22), the summation is across all $n$ - including any complex values of $\gamma_{n}$.

Finally, the study of wave propagation in waveguides of the class considered herein often involves the derivation of a power balance. Clearly, for the ducts shown in figures 1 and 2, there are two mechanisms by which energy propagates: through the fluid and along the flexible boundary. Given that the fluid velocity potential is of the form (7), a neat and convenient expression for the (non-dimensional) energy flux 
across an arbitrary vertical surface in a duct bounded above by an elastic plate and below by a rigid surface is

$$
\mathcal{E}=\frac{1}{\alpha} \sum_{n=0}^{N-1}\left|A_{n}\right|^{2} s_{n} C_{n}
$$

where $N$ is the number of "cut-on" modes. This expression, which was utilised by Warren et al [9] for a membrane bounded duct, incorporates both the fluid and the structure-borne components of energy flux and can be derived using the approach described by Crighton and Oswell [30] together with the appropriate OR.

\section{Discussion}

In this article a brief overview of the general theory underpinning a well known class of OR has been presented. Such ORs find application in a wide range of applications involving wave propagation in 2D (and lately 3D [27]-[29]) ducts or channels in the fields of both hydrodynamics and acoustics. The author has included a selection of references that demonstrate the versatility of the theory: wave propagation in structure/ice-covered water [11]-[18]; the study of acoustic silencers [21]-[23] and problems involving layers of distinct acoustic/fluid media [21]-[26]. The list of references is extensive but far from exhaustive.

Three points spring to mind. Firstly, Amaouche's and Meziani's papers [1, 2] do not seem to give due reference to the extensive and widely-published developments that have taken place in the last 12 years. Secondly, whilst noting that the first appearance of an OR of this class does appear to be due to Amaouche et al [3], in the light of the extensive development of the theory since then it seems inappropriate to refer to the OR of $[1,2]$ as being "new". Amaouche and Meziani [1, 2] investigate a hydroelastic system comprising two superposed fluids. Thus, as in (16), the eigenfunctions have a piecewise definition

$$
Y_{n}(y)= \begin{cases}Y_{1 n}(y), & -h_{1} \leq y<0 \\ Y_{2 n}(y), & 0<y \leq h_{2}\end{cases}
$$

and the appropriate OR is stated as

$$
\begin{aligned}
\omega^{2} \int_{-h_{1}}^{0} Y_{1 m} Y_{1 n} d y+\omega^{2} \rho \int_{0}^{h_{2}} Y_{2 m} Y_{2 n} d y & +\sigma_{1} Y_{1 m}^{\prime}(0) Y_{1 n}^{\prime}(0) \\
& +\rho \sigma_{2} Y_{2 m}^{\prime}\left(h_{2}\right) Y_{2 n}^{\prime}\left(h_{2}\right)=a_{n} \delta_{m n},
\end{aligned}
$$

where the reader is referred to $[1,2]$ for definitions of the parameters $\omega, \rho, \sigma_{j}, j=1,2$ and $a_{n}, n=0,1,2, \ldots$. On comparison of (17) and (25), it is clear that the latter is simply another example belonging to the class of OR discussed herein. The presence of an additional constant term on the left hand side of (25) arises due to the fact that both the fluid surface and fluid/fluid interface are described by a high order boundary condition. Again this is not new. Finally, in view of the close similarities between the two recent articles $[1,2]$ it would have been helpful to cross-reference them. 


\section{References}

[1] Amaouche M, Meziani B (2008) Oscillations of two superposed fluids in an open and flexible container, CR Mecanique, 336:329-335.

[2] Amaouche M Meziani, B (2011) Coupled frequencies of a rectangular hydroelastic system with two fluids, Meccanica, DOI 10.1007/s11012-010-9419-4.

[3] Amaouche M, Peube JL, Loraud JC (1981) Contribution à l'étude des ondes gravitocapillaires forcées d'amplitude finie, J Appl Math Phys, 32(4):382-395.

[4] Lawrie JB, Abrahams ID (2007) A brief historical perspective of the Wiener-Hopf technique, J. Eng Math, 59:351-358.

[5] Lawrie JB, Abrahams ID (1999) An orthogonality condition for a class of problem with high order boundary conditions; applications in sound/structure interaction, Q. Jl Mech appl. Math., 52:161-181.

[6] Andronov IV, Belinskii BP (1990) On acoustic boundary-contact problems for a vertically stratifed medium bounded from above by a plate with concentrated inhomogeneities, J. Appl. Math. Mech., 54:366-371.

[7] Belinskii BP (1989) The basisity of the system of eigenfunction of the transverse crosssection of waveguides with flexible wall, U.S.S.R. Comput. Maths. Math. Phys., 29:51-60.

[8] Warren DP(1999) The scattering of sound waves in two-dimensional ducts with discontinuites in height and material property. Dissertation, Brunel University, U.K.

[9] Warren DP, Lawrie JB, Mohamed IM (2002) Acoustic scattering in wave-guides with discontinuites in height and material property Wave Motion, 36:119-142.

[10] Kaplunov Y D, Kirillova IV, Postnova, YA (2004) Dispersion of waves in a plane acoustic layer with flexible elastic walls, Acoustical Physics, 50:694-698.

[11] Teng B, Cheng L, Lui SX, Li FJ (2001) Modified eigenfunction expansion methods for the interaction of water waves with a semi-infinite elastic plate, Appl Ocean Res, 23:357-368.

[12] Evans DV, Porter R (2003) Wave scattering by narrow cracks in ice sheets floating on water of finite depth, J. Fluid Mech. 484:143-165.

[13] Linton CM, Chung H (2003) Reflection and transmission at the ocean/sea-ice boundary, Wave Motion, 38:43-52.

[14] Chung H, Linton CM, (2005) Reflection and transmission of waves across a gap between two semi-infinite elastic plates on water, Q. Jl Mech appl. Math., 58:1-15.

[15] Manam SR, Bhattacharjee J, Sahoo T (2006) Expansion formulae in wave structure interaction problems, Proc. R. Soc. Lond. A., 462:263-287. 
[16] Porter R , Evans DV (2006) Scattering of flexural waves by multiple narrow cracks in ice sheets floating on water, Wave Motion, 43:425-443.

[17] Chung H, Fox C (2009) A direct relationship between bending waves and transition conditions of floating plates, Wave Motion, 46:468-479.

[18] Karmakar D, Bhattacharjee J, , Sahoo T (2010) Oblique flexural gravity-wave scattering due to changes in bottom topography, J. Eng. Math. 66:325-341.

[19] Lawrie JB (2007) On eigenfunction expansions associated with wave propagation along ducts with wave-bearing boundaries, IMA Jl Appl. Math., 72:376-394.

[20] Mohapatra SC, Sahoo T (2011) Surface gravity wave interaction with elastic bottom, Appl Ocean Res, 33:31-40.

[21] Mohamed-Guled IM, Lawrie JB (2003) Acoustic transmission through a silencer with wave-bearing boundaries, International Seminar: Day on Diffraction 2003, Proceedings, 130-141. IEEE Publications. DOI: 10.1109/DD.2003.1278245.

[22] Guled IMM (2003) Acoustic transmission: waveguides with scattering or dissipative components. Dissertation, Brunel University, U.K.

[23] Lawrie JB, Guled IMM (2006) On tuning a reactive silencer by varying the position of an internal membrane, J. Acoust. Soc. Am., 120:780-790.

[24] Bhattacharjee J, Sahoo T (2008) Flexural gravity wave problems in two-layer fluids, Wave Motion, 45:133-153.

[25] Mohapatra SC, Karmakar D, Sahoo T (2011) On capillary gravity-wave motion in two-layer fluids, J. Eng. Math. DOI 10.1007/s10665-011-9451-y

[26] Mahmood-Ul-Hassan, Meylan MH, Peters MA (2009) Water-wave scattering by submerged elastic plates Q. J1 Mech appl. Math., 62:321-344.

[27] Lawrie JB, Abrahams ID (2002) On the propagation and scattering of fluid-structural waves in a three-dimensional duct bounded by thin elastic walls. In: Abrahams ID et al (eds) Proceedings of IUTAM 2000/10. Fluid mechanics and its applications 68:279-288 Kluwer. ISBN 1-4020-0590-3.

[28] Lawrie JB (2009) Orthogonality relations for fluid-structural waves in a 3-D rectangular duct with flexible walls, Proc. R. Soc. A., 465:2347-2367.

[29] Lawrie JB (2010) Acoustic propagation in 3-D, rectangular ducts with flexible walls. In: INCE Conference Proceedings, 220(1):1930-1938. Institute of Noise Control Engineering of USA.

[30] Crighton DG, Oswell JE (1991) Fluid loading with mean flow. I. Response of an elastic plate to localized excitation, Phil. Trans. R. Soc. Lond. A 335:557-592. 